\begin{tabular}{|c|c|c|c|}
\hline $\begin{array}{l}\text { F O U N D A T I O N S } \\
\text { Vol. } 39\end{array}$ & $\begin{array}{c}\text { C O M P U T I N G A N D } \\
(2014)\end{array}$ & D E C I S I O N & $\begin{array}{r}\text { S C I E N C E S } \\
\text { No. } 1\end{array}$ \\
\hline DOI: $10.2478 /$ fcds-2014-0004 & & & $\begin{array}{l}\text { ISSN } 0867-6356 \\
\text { ISSN 2300-3405 }\end{array}$ \\
\hline
\end{tabular}

\title{
DYNAMICS OF INNOVATION DIFFUSION WITH TWO STEP DECISION PROCESS
}

\author{
Michał Szymczyk, Bogumił Kamiński*
}

\begin{abstract}
The paper discusses the dynamics of innovation diffusion among heterogeneous consumers. We assume that customers' decision making process is divided into two steps: testing the innovation and later potential adopting. Such a model setup is designed to imitate the mobile applications market. An innovation provider, to some extent, can control the innovation diffusion by two parameters: product quality and marketing activity. Using the multi-agent approach we identify factors influencing the saturation level and the speed of innovation adaptation in the artificial population. The results show that the expected level of innovation adoption among customer's friends and relative product quality and marketing campaign intensity are crucial factors explaining them. It has to be stressed that the product quality is more important for innovation saturation level and marketing campaign has bigger influence on the speed of diffusion. The topology of social network between customers is found important, but within investigated parameter range it has lover impact on innovation diffusion dynamics than the above mentioned factors.
\end{abstract}

Keywords: innovation diffusion, small-world networks, multi-agent simulation

\section{Introduction}

The aim of this paper is to investigate how the innovation spreads in the heterogeneous customers population. Traditional approach to this problem assumes that a person can learn about the innovation by communicating with other people or from the advertisements. Bass [2] studied the innovation diffusion using different communication channels. He divided the process of communication into two parts: (a) external influence of mass-media and advertising and (b) internal influence of the word-of-mouth processes. The word-of-mouth processes depend on the number of previous adopters and can be defined as passing the information between people by oral communication.

The problem of innovation diffusion is very important in economics and marketing. The success of innovation mainly depends on the long term saturation level and the speed of diffusion. When one defines the innovation as a product or a service, this success means the

* Szkoła Główna Handlowa, al. Niepodległości 162, 02-554 Warszawa, e-mail: bkamins@sgh.waw.pl 
real financial profits for the provider. However the speed of the diffusion is also an important factor, see for example [10]. A product that has very slow adoption rate might be abandoned by the producer in early stages even if it would have potentially high saturation level.

The word-of-mouth processes are often supported by marketing efforts. They allow to create the interest around the innovation and induce people to discuss about it. An innovation provider usually tries to control the roots of word-of-mouth processes and manipulate the innovation interest in the society. Nowadays, modern technologies such as internet offer large variety of possible activities, like discussions on blogs, forums, discussion groups, search engine optimizations (SEO), etc. The social networks services are very important word-of-mouth processes channels as they copy the interactions between people from the real world to the internet.

The word-of-mouth processes as a part of the innovation spread model were proposed by Bass [2]. He assumes that all consumers contact with themselves with the same intensity. This assumption, however, does not represent reality well. In a classic work on diffusion of innovations Rogers [8, p. 293] points out that "the heart of the diffusion process is the modeling and imitation by potential adopters of their near-peers". In this article we assume that social network of contacts between consumers society can be better represented using small-world topology [9]. Such a network is represented as a graph where the vertices are interpreted as consumers (agents) and its edges as the connections between them. Small-world networks combine two properties that are empirically validated: short average path length between clients and high average clustering coefficient [9].

The innovation diffusion model based on small-world network was proposed by Delre [4]. There, following Bass [2], it is assumed that the decision of adopting the innovation is made in a single step. Such a model is reasonable for traditional goods and is typically considered in models, see [8] for a review of state of simulation modeling approaches used in the literature. In e-commerce two-step decision-making process seems to be more popular. Most of the internet-based services are entirely or partially free or offer short-term free trials. In such cases a client can test the service without further commitments. If the client likes it, he or she decides to buy it. In such a situation clients make decision in twosteps:

1) to try the innovation or not;

2) to adopt (buy) the innovation or not, in case of earlier trial.

Such an approach based on the free trials is reasonable in industries where the cost of passing the product to the consumer is very low. In this case, if the company is convinced of the high quality of its product, it will be likely to make a high marketing effort to promote free version. This approach is similar to model of innovation-decision process proposed by [10].

Simulation in the model assumes that just one innovation spreads in the social network. First decision (to test the innovation or not) depends on marketing effort and innovation prevalence among friends. A consumer does not know anything about the quality of the innovation before trying - he just knows if his friends are using it. The second decision (to adopt the innovation or not) depends on the quality of the product and again on the innovation prevalence but to different extent. Then the consumer is immune to marketing influence because he tested the innovation by himself. The friends influence is still vital, because (apart from the recommendation effect) consumers often score higher utility levels 
when they use the internet-based services together with their friends. An example of such a case are network games or social networking services.

The trial of the innovation without adoption does not have any influence on the other agents in the network. There are two arguments for such an assumption. Firstly, a consumer can see among his friends only two groups of people: those who use the innovation and those who do not use it. In the first group there are consumers who have already adopted the innovation. In the second group there are both consumers who did not try the innovation and the ones who just tried the innovation without adopting it. The motivation for such a distinction is that a single consumer does not see the trials of the innovation but only its actual active usage. Moreover, agents usually will be less likely to communicate between each other about the trials than about successful adoptions. The second argument for the assumption is the model simplicity. It is not clear how the agents who tried the innovation might influence the newbies. On the one hand, their influence might be negative. One can consider the lack of adoption as the evidence of a bad product. On the other hand, agents who tested the innovation might simply wait for the others. They are ready to adopt it once they feel the social pressure strong enough.

Using the model we will try to deal with following research problems:

1. How do the product quality and marketing campaign intensity influence the innovation diffusion?

2. Are the social network topology and consumers susceptibility to peers influence important in the innovation diffusion?

The answer would help companies to balance the costs of these two investments in the product.

In particular we will verify the assertion that the bigger personal network of friends, the higher the probability that a consumer will learn about the innovation what should increase the speed of the diffusion. Especially, the model of Delre [4] showed the size of personal network is not a significant factor of innovation diffusion speed, but this process is the fastest in small-world networks. High susceptibility to friends influence of a consumer should foster the innovation diffusion. Delre [4] proved that in regular and small-world networks diffusion dynamics does not depend on this effect and that in random networks the speed of diffusion is faster in case of more individualistic society. It is the contradiction to the reasoning presented above. The question we answer in this paper is whether the results of Delre [4] would be preserved in two step decision-making process.

The article is divided into two sections. First, we present the problem of innovation diffusion and specification of two step decision-making process. Then we outline the specification of the simulation experiment and its results.

\section{Specification of two step decision making process}

In Section we describe the economic assumptions founding the model design and in Section we formalize them. Finally in section we outline the steps taken in the simulation procedure. 


\subsection{Assumptions about the diffusion dynamics}

Traditional diffusion models, based on epidemic models, assume that all the people are susceptible in the same way during the diffusion. Consumers decide to adopt the innovation in dependence on their attitude to the product and their friends behavior [6]. Delre [4] provides an example of cellular phones: almost everyone nowadays has a mobile phone, therefore there is a strong social pressure to have one. Even if someone were skeptical about the innovation, social pressure may convince them later to adopt it.

This reasoning leads to the term of critical mass. It was introduced by Rogers [10] and assumes some threshold value of adopters of innovation. Each consumer has a personal threshold and after surpassing this value, consumer becomes susceptible to the friends behavior. Thus critical mass concept uses the assumption of the feedback from the social network of the consumer.

This paper presents a more general approach to the diffusion dynamics problem. The main change is introduced by a two-step decision making process of the consumer. First of all, he decides to test the innovation or not. In case of earlier trial, he decides to adopt it or not. Such a distinction is a new approach to the innovation diffusion problem highlighting the difference between innovation usage and innovation trials.

Many businesses, especially the internet ones providing free services, face the problem of dead accounts. Some people try the service, create an account, look around and quit. On the one hand, they are listed as users and increase the accounts number. On the other hand, they do not use the service at all, so their account is useless for the service provider except they may boast with the theoretical number of accounts. Of course, it is possible that one day such a user will come back and start using the service - for example because many other people started using it and the fraction of adopters in his personal network surpasses the critical mass. As explained in the Introduction in the model proposed in this paper the group of testers is skipped in considerations of social influence. A consumer only sees whether his friends use the innovation or not.

Delre [4] assumes that a consumer can take part in innovation adoption decision only in case of at least one of his friends has already adopted the innovation. In this model there is no such a limitation. Potentially, agent can be convinced to test or adopt the innovation without the friends influence, just by the influence of product marketing.

There are two parameters of the innovation that provider can use to control the diffusion: product quality and product marketing. Quality is an abstract variable that corresponds with consumers' perception. There is an assumption that consumers are rational and they want as good product as possible. The value is in range from 0 - meaning completely useless and worthless product, to 1 - super quality product that provides everything that any consumer needs. There is nothing mentioned about the price of the product but this can be included into quality consideration. If someone wants to discuss the cost of obtaining the innovation, he simply needs to interpret quality parameter as the quality/price rate. Product marketing is an external product marketing that tries to convince a consumer to try the innovation. Again, the higher value, the more likely the consumer will be convinced to test the innovation. There is no discussion about the negative effects of the over-advertised product. The value is in range from 0 - meaning no advertising at all, to $1-$ no better marketing campaign is possible. 
The decision to test is based on two variables: product marketing and friends influence. Before trying, consumer does not know anything about the quality of the product, so this parameter is skipped. These two values are balanced using agent's parameter - marketing exposure. The higher this value, the less influential friends are and consumer is more susceptible to marketing campaigns. We assume that only adopters in consumer's personal network can influence him. The ones who tested do not have any influence, because they do not spread such a piece of information. Agents beyond customers personal network do not influence him at all because simply he does not have any information from them. Friends influence applies the critical mass concept. Product marketing has a linear effect on the consumer.

Product marketing and friends influence are balanced using marketing exposure parameter create the utility value for testing. Such a value is compared with minimum utility for testing which is a half of consumers minimum utility value. The reason of lowering the minimum utility in this decision is that it is easier to convince a person to try something than to adopt (buy) it - consumers more gladly try or taste something new, even if it is not in their taste or if they do not really need that. If the utility value surpasses the minimum utility, then agent decides to test the innovation.

The decision to adopt an innovation is based on two variables: product quality and friends influence. Such a decision is made only in case of earlier innovation trial. In this decision, product marketing is skipped. There is an assumption that after testing the consumer is resistant to advertisements and takes a rational decision without marketing influence. The reasoning is that if the innovation is useless for him, he will not use it only because the advertisements say so. These two values are balanced with agent's parameter quality preference. The higher this value, the less influential friends are and consumer is more focused on the product itself and its potential benefits. Friends influence scheme is the same like in decision of testing. Product quality has linear effect on the consumer.

Agents population creates a society - the connections between them are represented by a graph where vertices represent consumers and edges define the connections. Following Delre [4] we model this graph as a small-world network using Watts-Strogatz algorithm $[11]^{1}$. At the beginning of the simulation, set of initial innovators (that adopted the innovation) is chosen randomly. Then, for certain set of parameters, the simulation is run. For every single step, each agent is asked to test or adopt the innovation.

The next section presents the formal description of the model based on the above assumptions.

${ }^{1}$ Standard literature also considers algorithm proposed by Albert and Barabási [1] as a model of social interactions. It has a desirable property that it produces scale-free graphs. However, it has a shortcoming that it does not yield graphs with high level of clustering - which is an empirical phenomenon important in study of innovation diffusion, see for example [7]. Because of this shortcoming we have chosen to use Watts-Strogatz algorithm similarly to Delre [4]. 


\subsection{Specification of the innovation diffusion model}

Following [12] we identify three classes of variables driving innovation diffusion dynamics: characteristics of innovations, characteristics of innovators and environmental context. Therefore in our model setup we introduce set of 13 parameters:

A 2 parameters describing the product;

A 3 parameters describing the network (links between the consumers);

A 4 parameters describing the individual characteristics of agents;

A 4 technical parameters describing the simulation.

Below we present detailed interpretation of these parameters. Their range of values is discussed in Section 3.

There are two parameters of the product:

A $p q$-product quality - is an abstract value of the quality of the product; value in range of $[0 ; 1]$; the higher the value, the higher the quality;

A $p m$ - product marketing - is the external marketing effort; value in range of $[0 ; 1]$; the higher the value, the more influential marketing campaign;

Network is described by three parameters:

A $r p$-rewire probability - this is the parameter $p$ in Watts-Strogatz [11] model and defines the level of randomness of the network; value in range of [0;1]; where 0 regular network, 1 - random network;

A $f_{0}$ - order of friends range - describes the extent of personal network for each agent; direct friends are first alters $(f o=1)$, friends of friends are second alters $\left(f_{o}=2\right)$ and so on; this parameter does not have to be integer; if it is real, for example $f_{o}=1.5$, it means that first alters are counted normally, but the second alters have only $50 \%$ of their influence (it is vital that firstly direct friends are counted and later further friends - there is a mechanism of avoiding double counting the same friends, this is similar to breadth-first search instead of depthfirst search); the value is not smaller than 1 ;

A $f n$ - friends number - the average number of direct friends in the network; in regular network all the agents have the same number of direct friends, in case of random networks, individual number of links is not constant.

Agent characteristics are described by four parameters:

A me - marketing exposure - weight the marketing against friends' influence in decision of testing the innovation; value in range of $[0 ; 1]$; the higher the value, the more susceptible on marketing the agent is and friends have lower influence on him;

A $q p$ - quality preference - weights the quality and friends' influence in decision of adopting the innovation; value in range of $[0 ; 1]$; the higher the value, the more important quality of the product is and friends have lower influence on agent;

A $m u$ - minimum utility - mean minimum utility requirement, common for decisions of testing and adopting; the higher the value, the harder to convince an agent to test or adopt the innovation; value in range of $[0 ; 1]$;

A $f t$ - friends threshold - mean fraction of friends that adopted the innovation treated as critical mass level; for decision of testing the threshold is a half of $f t$; value in range of $[0 ; 1]$; this value is compared with a fraction of friends in the personal 
network (defined by the parameter fo) who adopted the innovation; if and only if the threshold is surpassed, then friends do have an influence on the decision.

The above parameters define a scenario for the simulation. Moreover, there are four constants that complete the scenario of the specification of simulation:

A $n a$-number of agents in a single network;

A $n n$ - number of networks for one scenario; for each set of parameters (of product, network and agents), there are $n n$ networks generated and the results are averaged - such a procedure decreases the variance of results;

A $s s$ - number of steps in single simulation;

A in - number of innovators - an innovator is an agent that adopted the innovation before starting the simulation;

Now we will provide formal description of the agents decision-making process.

Agent decides to test the innovation, when

$$
u T \geq m u
$$

where $m u$ - agent's minimum utility, and

$$
\begin{gathered}
u T=m e \cdot p m+(1-m e) \cdot f i t \\
f i t=\left\{\begin{array}{lc}
1, & \text { if } \quad f r \geq 0.5 \cdot f t \\
0, & \text { otherwise }
\end{array}\right.
\end{gathered}
$$

where $f r$ - fraction of friends in $f o$ order who adopted the innovation, $m e$ - marketing exposure, $p m$ - product marketing, fit - friends' influence for testing.

Agent decides to adopt the innovation, when

$$
u A \geq m u
$$

where $m u$ - agent's minimum utility, and

$$
\begin{gathered}
u A=q p \cdot p q+(1-q p) \cdot f i a \\
f i a= \begin{cases}1, & \text { if } \quad f r \geq f t \\
0, & \text { otherwise }\end{cases}
\end{gathered}
$$

where $f r$ - fraction of friends in $f o$ order who adopted the innovation, $q p$ - quality preference, $p q$ - product quality, fia - friends influence for adoption.

If the utility function surpasses personal threshold, the innovation is respectively tested or adopted.

Product marketing and quality have linear effect on the agent (weighted respectively by marketing exposure and quality preference against friends influence). Even a small value of them influences agent's decision. With social influence it is different. There is a threshold introduced - if the fraction of adopters in agent's personal network surpasses the threshold then the agent does feel social influence, otherwise not. Granovetter [5] argues that a consumer does not feel influence from just a few friends behaving in some way. But when these people reach a certain critical number, the consumer starts imitating them. 
The possible states and transitions between them are presented in In each step of the simulation each agent is asked if he wants to try (or adopt) the innovation or not. It means that in each step of the simulation any agent can change their state or remain in the current one. Only the agents who have already adopted the innovation cannot change their state. For example, an agent might not want to try the innovation for several simulation steps. But the social pressure might increase and after some time he might be convinced to try it. Similarly, he might not want to adopt the innovation ad hoc, but rather wants to wait for more friends around him using the innovation. In a single step, all the agents take their decisions in exactly the same moment under exactly the same conditions (so called synchronous updating is applied).

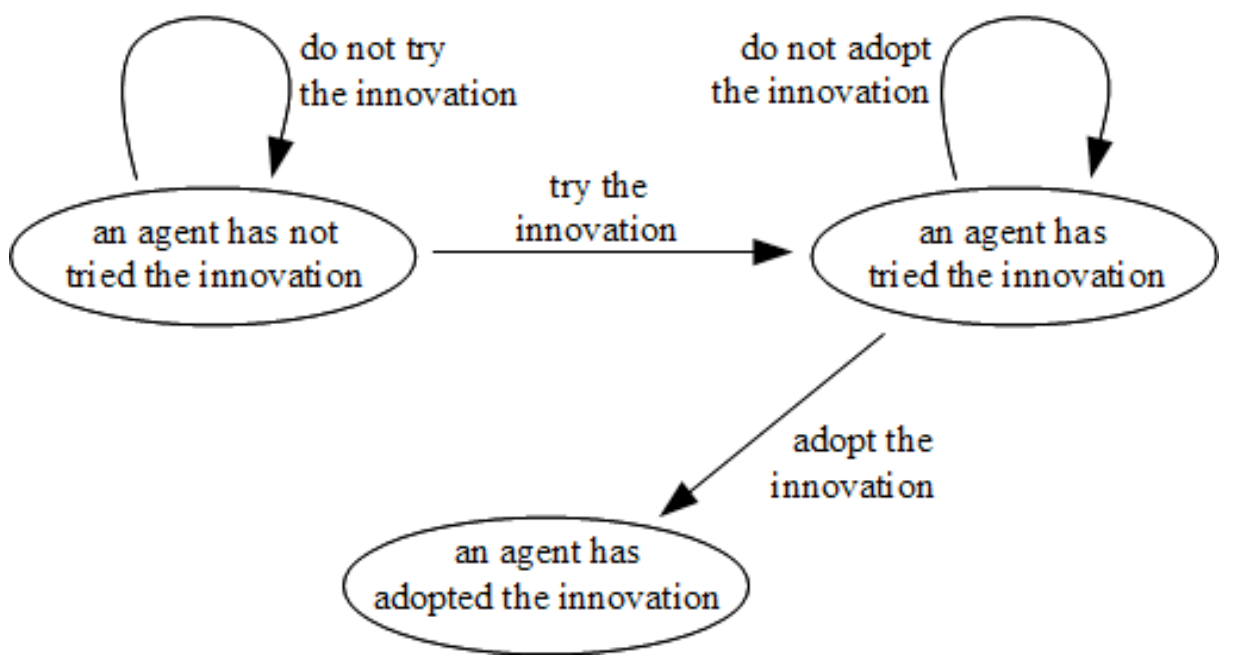

Figure 1. Possible states (represented as ellipses) of an agent and possible transitions (represented as arrows) between the states. In each simulation step an agent does exactly one movement. All agents except the innovators are in the first state at the beginning what means they have not tried the innovation. The innovators are in the last state what means they have adopted the innovation.

Each simulation finishes with two outcomes:

A saturation level - equals the number of adopter divided by number of all agents in a network; it is never zero because at the beginning there is a group of innovators who adopted the innovation;

A time between $0 \%$ and $75 \%$ of final saturation - how much time it takes to reach $75 \%$ of final saturation

The first outcome - saturation level - describes the long run level of the innovation adoption in the population of agents. The second outcome measures the speed of the innovation diffusion. Its measurement is terminated at the level of $75 \%$ of final saturation because the aim is to focus on the initial period after introduction of the innovation. This parameter is crucial for the company to conclude at the beginning whether to continue investments in the innovation or not. Terminating the measurement at the level of $75 \%$ results in skipping the stragglers [10]. Other than $0 \%$ to $75 \%$ intervals were tested and they produced similar results. 


\subsection{The description of the model simulation stages}

For each scenario run there is one network generated. Network generation is divided into four parts:

1) creating agents,

2) generating regular network,

3) randomizing the network,

4) choosing the innovators.

At the beginning, the set of agents is created using the scenario parameters. The regular network generation is the first step of Watts-Strogatz model algorithm and it uses the $f n$ parameter. If the $r p$ parameter is greater than zero, then network is randomized - following the second step of Watts-Strogatz model algorithm. When all selected edges of the graph are rewired, the innovators are drawn randomly. At the end, for each agent we count number of friends (constant during the simulation), friends who tested and who adopted (variable during the simulation). These three values are counted depending on fo parameter which tells about the private network extent. Such a network is ready for the simulation.

Simulations are run in parallel. The software application developed to perform it uses the multi-threading mechanism. This allows to speed up the calculations and take the advantage of multi-core CPUs.

For each set of network and agents parameters, $n n$ single simulations are run. The results are averaged and saved as a single line in an output file. Each line contains the values of parameters and outcome variables.

A single simulation carries out the $s s$ steps. After each step the number of adopters is saved (it helps calculating the dynamics of diffusion). In case everyone adopted the innovation, the remaining steps are skipped.

In each step of the simulation, each agent is asked to take a decision:

A to test the innovation or not, if he has not already tested it - in accordance with the formulas (1), (2) and (3)

A to adopt the innovation or not, if he has already tested it, but has not adopted it yet - in accordance with the formulas (4), (5) and (6)

Then for each agent the numbers of friends who tested and adopted are updated. Such a procedure guarantees that the situation does not change in a single step, and that all decisions in a single step are taken in the same conditions.

In the next section we present the design of simulation experiment and the conclusions that can be drawn from it.

\section{Simulation parametrization and results analysis}

The simulation was run for 17500 sets of random parameters. Every time, the parameters were drawn uniformly, in accordance with Because of the stochastic nature of the simulation, for each parameters set, there were $n n=32$ runs and the average outcomes values were recorded. The time of a single simulation was constant $s s=150$. The value of this parameter was set empirically as high enough to reach the saturation.

The measured outcomes are:

A percentage level of innovation saturation in long term: $s$; 
A the time required to reach $75 \%$ of final saturation: $t$.

The long term saturation level $s$ is measured as a variable of three values, in accordance with a. The low level of variable $s$ is selected so as to reflect the fact that the network is always initially seeded with innovators. The time required to reach $75 \%$ saturation $t$ is defined as a binary variable, in accordance with $\mathrm{b}$. Variable $t$ is measured only in case of the saturation level higher than $5 \%$ (so when the innovation saturation is at least medium).

The analysis of simulation results is carried out in two steps. Firstly, we build a model that identifies the predictors of innovation adoption. The lack of innovation adoption is understood as the value of outcome $s$ equal to low. In case of innovation adoption we discuss whether the innovation is adopted on the medium or high level (dependent variable $s$ ) and whether this process if fast or slow (dependent variable $t$ ).

The data analysis was carried out using logistic regression and random forests [3]. Logistic regression was used to state the direction of influence of the independent variables to the dependent variables. Random forest were better in prediction and they were used to identify the most important variables explaining the diffusion process.

Table 1. Distributions used in drawing the simulation parameters

\begin{tabular}{|l|l|c|}
\hline \multicolumn{1}{|c|}{ Parameter } & \multicolumn{1}{c|}{ Code } & $\begin{array}{c}\text { Range of } \\
\text { changeability }\end{array}$ \\
\hline \multicolumn{3}{|c|}{ Product parameters } \\
\hline Product quality & $p q$ & {$[0.1 ; 0.9]$} \\
\hline Product marketing & $p m$ & {$[0.1 ; 0.9]$} \\
\hline \multicolumn{2}{|c|}{ Network parameters } \\
\hline Rewiring probability & $r p$ & {$[0 ; 1]$} \\
\hline Range of personal network & $f o$ & {$[1 ; 2]$} \\
\hline Friends number & $f n$ & $8,4,6\}$ \\
\hline Innovators number & $i n$ & 512 \\
\hline Number of agents in a network & $n a$ & {$[0.1 ; 0.9]$} \\
\hline & $n a$ & {$[0.1 ; 0.9]$} \\
\hline Minimum utility & $m u$ & {$[0.1 ; 0.9]$} \\
\hline Friends threshold & $f t$ & {$[0.1 ; 0.9]$} \\
\hline Marketing exposure & $m e$ & \\
\hline Quality preference & $q p$ & \\
\hline
\end{tabular}

Table 2. Definition of variable $s$ and $t$.

\begin{tabular}{|l|r|c|}
\hline \multicolumn{1}{|c|}{ Label } & Percentage & $\begin{array}{c}\text { Fraction of } \\
\text { adopters }\end{array}$ \\
\hline low & $73.5 \%$ & $<5 \%$ \\
medium & $9.5 \%$ & {$[5 \% ; 90 \%]$} \\
high & $17.0 \%$ & $>90 \%$ \\
\hline
\end{tabular}

(a) variable $s$

\begin{tabular}{|l|r|c|}
\hline \multicolumn{1}{|c|}{ Label } & $\begin{array}{c}\text { Percentage } \\
(s>\text { low })\end{array}$ & $\begin{array}{c}\text { Time to } 75 \% \\
\text { of saturation }\end{array}$ \\
\hline $\begin{array}{l}\text { fast } \\
\text { slow }\end{array}$ & $51.4 \%$ & $\leq 5 \%$ \\
& $48.6 \%$ & $>5 \%$ \\
\hline
\end{tabular}

(b) variable $t$

In the prediction models, apart from the raw simulation parameters, we defined two other variables: the level of product marketing divided by the minimum utility 
$\mathrm{pm} / \mathrm{mu}$ and the product quality divided by the minimum utility $p q m u=p q / m u$. Moreover variable $f n$ is analyzed as a nominal variable.

The first analysis is the identification of factors that determine the lack of innovation adoption. We investigate the variable $s=$ low (innovation was no adopted) against medium and high together. The results of the logistic regression estimation are presented in, the estimation of variables significance and the model quality is given in

Table 3. The results of logistic regression explaining the failure of the innovation (market value is $l o w)$.

\begin{tabular}{|c|r|c|r|c|r|}
\hline Variable & Parameter & Variable & Parameter & Variable & Parameter \\
\hline$p m m u$ & -0.40 & $r p$ & 0.84 & $m u$ & 5.49 \\
\hline$p q m u$ & -0.74 & $f o$ & 1.16 & $f t$ & 8.18 \\
\hline$p q$ & -1.82 & $f n=4$ & 0.80 & $m e$ & -0.64 \\
\hline$p m$ & -1.38 & $f n=6$ & 1.36 & $q p$ & -2.52 \\
\hline
\end{tabular}

Variable Importance

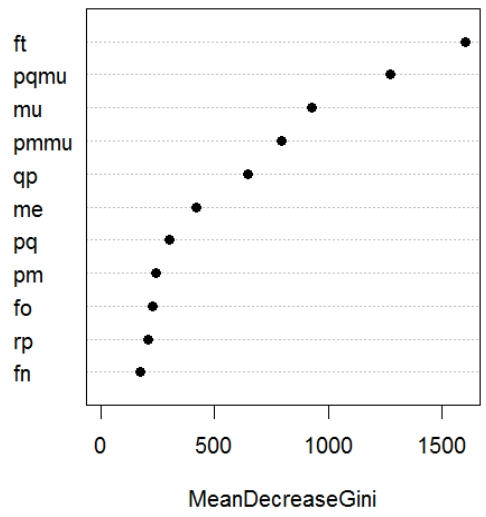

ROC Curve

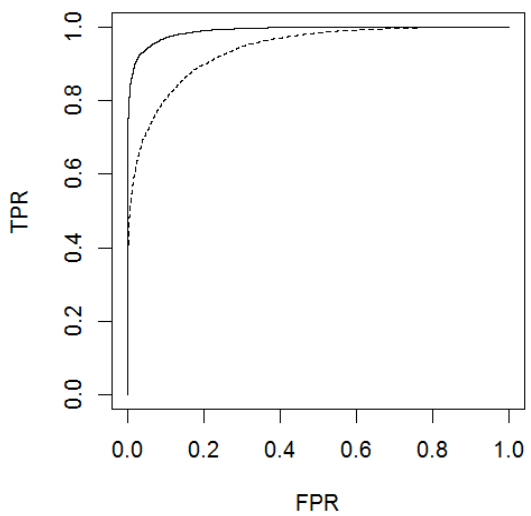

Figure 2. Estimation of the importance of variables describing the probability of no adoption of the innovation using the random forests, and comparison the prediction ability of random forests (solid line) and logistic regression (dashed line) using ROC curve.

First of all, in accordance with the expectations, the higher the product quality ( $p q$ and $p q m u)$ and the product marketing ( $p m$ and pmmu), the lower the risk of failure of the innovation. The more dispersed friends in the network are (high values of $r p, f o$ and $f n$ ) the higher the risk of failure. Taking into account agents' parameters, the higher their expectations of the product (variables $m u$ and $f t$ ), the lower the chance for the success - as was expected. The lower their susceptibility to the society (high values of variables me and $q p$ ) is more advantageous to the innovation.

Analysis of the variables importance leads to interesting conclusions. The most important factor influencing innovation adoption is the friends threshold $(f t)$. More important are parameters dealing with product quality (pqmu) rather than marketing $(\mathrm{pmmu})$. Also significant is the level of quality expectations $(\mathrm{mu})$. Relatively low is the importance of the variables describing the network of agents ( $r p, f o$ and $f n)$. One has to see 
that presented random forest model has very high prediction ability (especially, much higher than logistic regression). The area under the RUC curve (AUC) is $98.99 \%$ which means that with such a probability random observation with a label low has higher score value than randomly draw observation with a label medium or high. In other words, the model almost ideally separates the observations.

Now let us investigate determinants of the innovation success in case the innovation is adopted in the market. Such an approach is especially interesting, as we showed in previous model that it is possible to estimate the chance of adopting the innovation with very high precision.

First we present the results of analysis the level of innovation adaptation (medium against high). In one can find the results of estimation the direction of parameters influence using logistic regression. In the the importance of these variables calculated using random forests and the quality of obtained classifiers are given.

Observed results are very similar to the previous model (notice the change of the sign of the estimations, as in this model the marked value is high level of variable $s$ ). Looking into the differences between the relations, one has to see the change of direction of variable $f n$ influence. The higher its value the higher the probability of high level of innovation adoption. But it is the least important variable in the model. In the analysis of variables importance there is one more change - to have high level of innovation adoption, quality preference $(q p)$ is very important. Obtained quality of the classifier of random forest is high $(\mathrm{AUC}=93.35 \%)$.

Table 4. Results of logistic regression explaining the level of innovation adoption (marked value is high) in case of its success.

\begin{tabular}{|c|r|c|r|c|r|}
\hline Variable & Parameter & Variable & Parameter & Variable & Parameter \\
\hline$p m m u$ & 0.24 & $r p$ & -0.27 & $m u$ & -2.39 \\
\hline$p q m u$ & 0.46 & $f o$ & -0.43 & $f t$ & -4.22 \\
\hline$p q$ & 1.23 & $f n=4$ & 0.50 & $m e$ & 1.62 \\
\hline$p m$ & 0.75 & $f n=6$ & 0.30 & $q p$ & 2.70 \\
\hline
\end{tabular}

Variable Importance

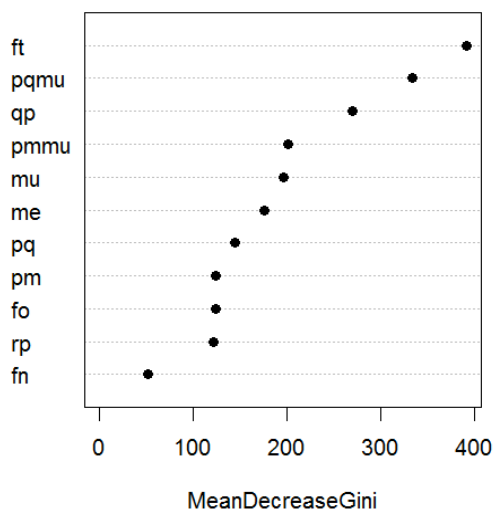

ROC Curve

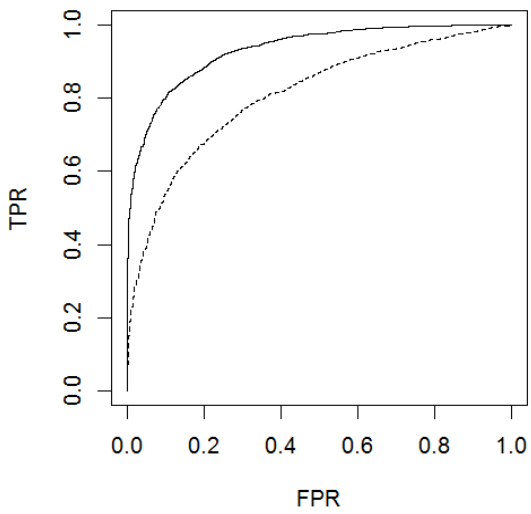

Figure 3. The estimation of variables importance in case of high level of innovation adoption when it is adopted at all using random forests, and comparison of prediction 


\section{ability random forest (solid line) and logistic regression (dashed line) using ROC curve.}

Now let us discuss the speed of innovation adoption. In the logistic regression model that estimates the probability that the innovation will spread slowly, two variables: $p q$ and $m u$ are irrelevant statistically. Comparing to the model where the innovation was a success () it is noticeable that high dispersion of consumers in the network increases the speed of innovation adoption (variables $r p, f o, f n$ ), similarly as increasing the friends threshold value $(f t)$. One has to bear in mind that it changes the set of the most important variables in the model. For the speed of innovation diffusion the most important are relative product marketing ( cases obtained model has very high prediction ability $(\mathrm{AUC}=94.92 \%)$.

Summing up, random forests helped to find a good explanation of the fact, level and speed of innovation adoption. The most important variables explaining these processes are friends threshold $(f t)$ and relative quality and intensity product marketing (pqmu and ртти). Product quality is more important when describing the level of innovation saturation and product marketing is more significant for the speed of innovation diffusion.

Table 5. The results of logistic regression explaining the speed of innovation adoption (marked value is slow) in case of its adoption.

\begin{tabular}{|c|r|c|r|c|r|}
\hline Variable & Parameter & Variable & Parameter & Variable & Parameter \\
\hline$p m m u$ & -0.79 & $r p$ & -1.08 & $m u$ & 0.00 \\
\hline$p q m u$ & -0.36 & $f o$ & -0.45 & $f t$ & -2.66 \\
\hline$p q$ & 0.00 & $f n=4$ & -0.75 & $m e$ & -4.35 \\
\hline$p m$ & -0.89 & $f n=6$ & -1.32 & $q p$ & -1.46 \\
\hline
\end{tabular}

Variable Importance

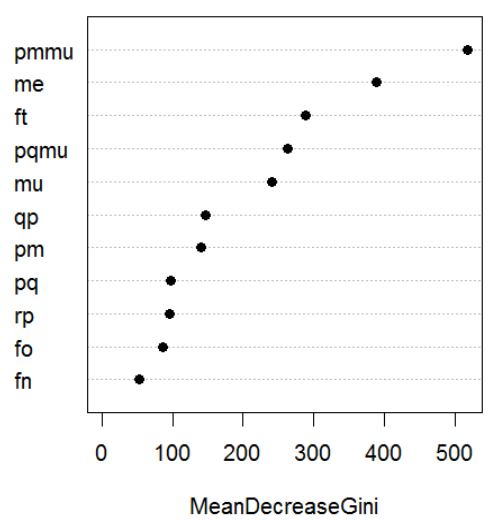

ROC Curve

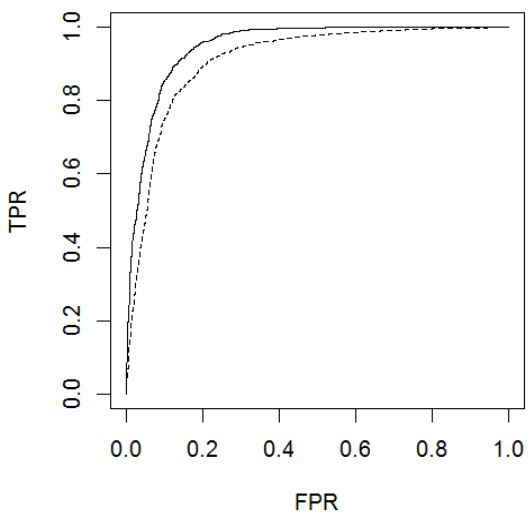

Figure 4. The estimations of variables importance describing the speed of innovation adoption in case of its success using random forests and comparison of prediction abilities of random forests (solid line) and logistic regression (dashed line). 


\section{Concluding remarks}

In this article, we investigated the innovation diffusion with heterogeneous consumers. The innovative part of this article in comparison to previous researches is two step decisionmaking process by consumers. Such a process separates testing and adopting the innovation. This can be observed more often nowadays in industries where cost of providing the service or product for testing is low. Typical examples are services offered in the internet.

In this article we identified the factors explaining the fact of innovation adoption and level and speed of its diffusion. The most important variables explaining this processes are the friends threshold level and relative product quality and intensity of product marketing. It means that in case of new product analysis it is very important to consider to what extent friends opinion is important. Such a typical products are social networks and on-line games. Satisfaction from using them is much higher when friends are using them as well.

It has to be noted that for the level of innovation saturation product quality is the most important company-dependent parameter. Product marketing is more influential on the speed of innovation diffusion. It is meaningful in planning of the strategy of new product introduction into the market. Intensive product marketing allows to verify whether the product will be adopted in the society as an innovation or it is a failure because of the quality that does not meet clients requirements.

The proposed model is theoretical in nature and thus can be used only to provide qualitative conclusions and give general recommendations. Further research can be carried out after acquiring data about the spread of a real product innovation in the internet and seeking for model parameterization that reproduces its diffusion.

\section{References}

[1] Albert R., Barabási A.-L., Statistical mechanics of complex networks, Reviews of Modern Physics, 74, 2002, 47-97

[2] Bass F. M., A new product growth for model consumer durables, Management Science, 15, 1969, 215-227.

[3] Breiman L., Random Forests, Machine Learning, 45, 2001, 5-32.

[4] Delre S. A., Jager W., Janssen M. A., Diffusion dynamics in small-world networks with heterogeneous consumers, Computational \& Mathematical Organization Theory (2007) 13, 185202.

[5] Granovetter M., Threshold models of collective behaviour, American journal of sociology, 83, 1978, 1420-1443.

[6] Granovetter M., Soong R, Threshold models of interpersonal effects in consumer demand, Journal of Economic Behaviour \& Organization, 7, 1986, 83-99.

[7] McCullen N. J., Rucklidge A. M., Bale C. S. E., Foxon T. J., Gale W. F., Multiparameter Models of Innovation Diffusion on Complex Networks, SIAM Journal of Applied Dynamical Systems, 12, 2013, 515-532

[8] Nan N., Zmud R., Yetgin E., A complex adaptive systems perspective of innovation diffusion: an integrated theory and validated virtual laboratory, Computational and Mathematical Organization Theory, Springer, May 2013, DOI: 10.1007/s10588-013-9159-9

[9] Newman M. E. J., The Structure and Function of Complex Networks, Society for Industrial and Applied Mathematics Review, 45, 2, 2003, 167-256. 
[10] Rogers, E. M., Diffusion of innovations (3rd ed.), Free Press, New York, 1983.

[11] Watts D.J., Strogatz S.H., Collective dynamics of "small-world" networks. Nature, 393, 1998, 40-442.

[12] Wejnert B., Integrating Models of Diffusion of Innovations: A Conceptual Framework, Annual Review of Sociology, 28, 2002, 297-306

Presented at the XII Conference: Systems and Operational Research - BOS 2012, 17-19 September 2012, Warsaw, Poland 